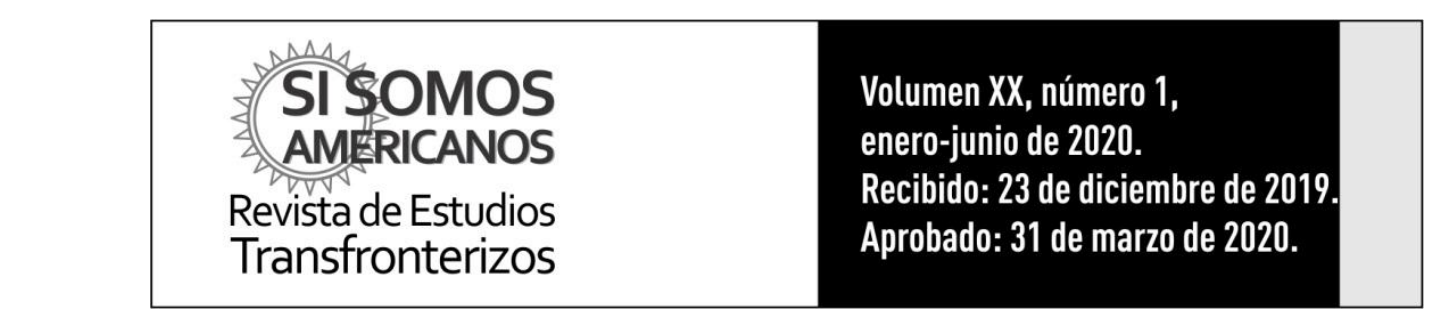

\title{
Formación interdisciplinaria, colaboración y mirada comparada en el estudio de la convivencia y el multiculturalismo cotidiano: una entrevista a Amanda Wise y Selvaraj Velayutham*
}

\section{Interdisciplinary training, collaboration and a comparative perspective on the study of conviviality and everyday multiculturalism: An interview with Amanda Wise and Selvaraj Velayutham}

\author{
Carol Chan** \\ Universidad Academia de Humanismo Cristiano, Chile. \\ Carolina Ramírez*** \\ Centro de Estudios de Conflicto y Cohesión Social (COES) \\ Universidad de Chile, Chile
}

\section{Resumen}

En la última década los estudios acerca de la convivencia y la multiculturalidad cotidiana han cobrado renovada importancia en los estudios étnicos, migratorios y urbanos. Esta entrevista recoge las voces de la investigadora australiana Amanda Wise, y su par

* Esta entrevista se enmarca en las actividades organizadas por la Red Internacional Migración, Etnicidad y Espacio: Aproximaciones Críticas desde la Etnografía (MES Network), la cual ha sido financiada por el Programa de Cooperación Internacional (PCI) de la Agencia Nacional de Investigación y Desarrollo (ANID) (anteriormente Comisión Nacional de Ciencia y Tecnología de Chile, CONICYT), (REDI170315). Además, cuenta con el patrocinio del Fondo Nacional de Desarrollo Científico y Tecnológico (FONDECYT, PROYECTOS N³160327 y 3170051), y el apoyo de COES (ANID/FONDAP/15130009).

** Bachelor of Arts (Antropología), University of Melbourne (Australia). Doctora en Antropología Cultural, University of Pittsburgh (EEUU). Universidad Academia de Humanismo Cristiano, Chile. Correo electrónico: carolchanzilin@gmail.com

*** Licenciada en Sociología, Pontificia Universidad Católica de Chile. Magister en Metodologías de la Investigación, Goldsmiths, Universidad de Londres. PhD en Sociología Visual, Goldsmiths, Universidad de Londres. Centro de Estudios de Conflicto y Cohesión Social (COES). Universidad de Chile, Chile. Correo electrónico: carolina.rmc@gmail.com

Cómo citar este artículo: Chan, C. y Ramíres, C. (2020). Formación interdisciplinaria, colaboración y mirada comparada en el estudio de la convivencia y el multiculturalismo cotidiano: una entrevista a Amanda Wise y Selvaraj. Si Somos Americanos. Revista de Estudios Transfronterizos, 20(1), 203-216. doi: 10.4067/S0719-09482020000100203 
singapurense Selvaraj Velayutham, quienes en solitario y en colaboración han generado notables aportes para el estudio de la experiencia de vivir juntos a través de la diferencia cultural en contextos urbanos diversos. En esta conversación, junto con discutir su marco conceptual, se recogen aspectos relativos a su formación, aproximación interdisciplinaria, y su trabajo colaborativo y comparado. Además, ambos esbozan reflexiones iniciales en torno a las particularidades de los procesos de diversidad y convivencia en la ciudad de Santiago; reflexiones basadas en observaciones y discusiones llevadas a cabo durante una estadía académica en Chile.

\section{PRESENTACIÓN}

Amanda Wise es académica en el Departamento de Sociología de Macquarie University, Sídney, Australia. Es particularmente conocida por su trabajo en ciudades globales y diversidad urbana comparada; 'multiculturalismo cotidiano' (everyday multiculturalism); raza y relaciones interétnicas; comunidades diaspóricas, transnacionales y migrantes; movilidad laboral en y desde Asia. Ha publicado numerosos artículos en estos temas y es autora del libro Exile and Return among the East Timorese (University of Pennsylvania Press, 2006) y coeditora (con Selvaraj Velayutham) de Everyday Multiculturalism (Palgrave, 2009). Se ha centrado en la materialidad, civilidad y sensorialidad de la vida urbana, y en la formación de 'comunidad' en diversos contextos de Asia y Australia. Su trabajo considera la mutual relación entre lo subjetivo, procesual y estructural, dimensiones que dan forma a las experiencias de vivir con diversidad.

Selvaraj Velayutham es académico el Departamento de Sociología de Macquarie University, Sídney, Australia. Es conocido por su contribución a los campos de migración internacional, multiculturalismo cotidiano, y sociología de la vida cotidiana, y en estudios culturales. Junto con contar con numerosos artículos en estos temas, es autor de Responding to Globalisation: Nation, Culture and Identity in Singapore (ISEAS-Yusof Ishak Institute, 2007), y coeditor (con Melissa Butcher) de Dissent and Cultural Resistance in Asian Cities (Routledge, 2009) y (con Amanda Wise) de Everyday Multiculturalism (Palgrave, 2009). En sus investigaciones explora como ideas acerca de raza y racismo son cotidianamente institucionalizadas y experimentadas por las personas en diferentes escenarios, o se manifiestan (como resistencia, apropiación cultural y otros mecanismos) en diferentes expresiones y productos culturales.

Ambos académicos han colaborado extensivamente en investigaciones acerca de multiculturalismo cotidiano y en artículos en coautoría acerca de experiencias de diversidad en Singapur y Australia. Durante su visita a Chile a fines del 2018 como investigadores asociados a la red Migración, Etnicidad y Espacio: Aproximaciones Críticas desde la Etnografía (ANID-PCI, REDI170315), quisimos entrevistarlos en conjunto. Estábamos especialmente interesadas en su formación como investigadores y las experiencias que 
influenciaron su foco y aproximación, así como también los desafíos y beneficios que surgen del trabajo colaborativo a largo plazo y que visualizan para su campo de estudios.

\section{ENTREVISTA}

\section{Experiencias y trayectorias}

Carolina Ramírez: ¿Podrían contarnos acerca de su background disciplinario y de cómo sus trayectorias académicas han dado forma al trabajo que han venido haciendo?

Selvaraj Velayutham: Mi formación en pregrado fue muy interdisciplinaria; tomé cursos ofrecidos por los departamentos de antropología, sociología, estudios culturales, en feminismo, medios, política, y economía política. Esto me condujo hacia mi trabajo doctoral el cual fue en sociología y estudios culturales. Creo los estudios culturales siempre han abrazado cierta diversidad de influencias disciplinarias, o al menos no son tan rígidos. Pero una limitación fueron los tipos de métodos que uno podría desarrollar. El más popular era análisis del discurso, textual o de políticas; pocas personas conducían entrevistas o trabajo de campo, y en cierto sentido eso moldeó el foco de mi investigación. Me pareció que el análisis del discurso y de la política no ofrecía una comprensión rica y matizada del impacto de la globalización en las experiencias de vida de las personas.

Y luego, para mi investigación postdoctoral en colaboración con Amanda (Wise), condujimos un estudio cualitativo acerca de migrantes laborales calificados temporales de India en Australia. Este estudio involucró observación participante, trabajo de campo y entrevistas. Fue entonces cuando comenzamos a reunir aproximaciones de la antropología, sociología, geografía cultural, estudios de género y otras. El background de Amanda en sociología y antropología, y su investigación acerca de los refugiados de Timor Oriental en Australia y 'zonas de contacto' en el barrio culturalmente diverso de Ashfield (en los suburbios de Sydney) influenciaron fuertemente mi mirada. Siendo una pareja conduciendo investigación en diferentes campos, eventualmente había convergencias. Me involucré mucho en ambos proyectos de Amanda, lo que influenció mi forma de pensar la conducción de un estudio, la ideación de preguntas de investigación, el uso de teorías y conceptos.

Amanda Wise: mi licenciatura fue en sociología, a inicios de los 1990s, cuando los híperpost-estructuralistas estaban infiltrándose en sociología. Al mismo tiempo, yo estaba vinculada con un profesor que era fuertemente interaccionista simbólico, y fui influenciada por ambas posturas. Me fasciné con los teóricos post-estructuralistas cuando me los presentaron, pero luego comencé a escribir acerca de derechos humanos para mi Honors thesis, que era acerca de la comisión de verdad y reconciliación en Sudáfrica, y me convertí en una derridiana, o algo más. Luego, simplemente llegué a un límite metodológicamente. 
Mientras hablaba de la materialidad de carne y hueso del trauma, desarrollé una aversión hacia aquella teoría tan abstracta. Así fue como giré a estudiar en profundidad el fenómeno de la vida cotidiana... y etnografía es mi modalidad de investigación preferida. De algún modo esto ha informado mis principales intereses de investigación desde entonces orientada a una aproximación más fenomenológica, con un interés en las dimensiones corporizadas y afectivas de la experiencia, ya sean experiencias prosaicas o las brutalidades de la guerra, el apartheid o el exilio.

Para mi doctorado trabajé en Timor Oriental, así que me moví de Sociología a un centro de investigación interdisciplinario llamado Center for Research for Intercommunal Studies. Ien Ang fue una de mis supervisoras y también de Raj (Velayutham)... quizás así convergía nuestra investigación en ese tiempo. Ella (Ang) era de la tradición Británica de la Escuela de Estudios Culturales de Birmingham y mi supervisora principal era Mandy Thomas, una antropóloga que estaba escribiendo acerca de exiliados vietnamitas. Mientras estábamos ahí, el centro recibió a personas como James Clifford, Pnina Werbner y Michael Herzfeld, y esas voces influenciaron nuestro trabajo, como también lo hizo Greg Noble, uno de los miembros fundadores (del centro) y con quien continúo colaborando. Mi tesis de doctorado acerca de refugiados de Timor Oriental fue publicada como libro (Wise 2006). Esta investigación abrió mi interés en afecto, con preguntas acerca de los refugiados y exiliados políticos. Esto me llevó a la literatura en teoría del trauma, y así llegué a estar realmente interesada en el terreno de lo sensorial y corporizado.

Luego me interesé en los simpatizantes que solidarizaban, en Australia, con la lucha por la independencia de Timor Oriental desde los 1970s a los 1990s. Estas redes de solidaridad estaban formadas por los usuales intelectuales de la ciudad, estudiantes y activistas, pero descubrí que quienes participaban en la campaña por la independencia (de Timor Oriental) eran apoyados por esta inmensa red de mujeres blancas mayores, que estaban basadas en iglesias católicas de pueblos y suburbios a lo largo de Australia, y ellas eran apoyadas (a su vez) por una orden de monjas Josephite. Estas mujeres hacían recaudación de fondos, escribían cartas a periódicos y hacían todo tipo de cosas. Desde allí generé un interés en solidaridades a través de las diferencias étnicas, y en experiencias vividas de multiculturalismo, y trabajo interétnico.

Esa investigación era también un tópico difícil. Estuve lidiando tres años con muchas personas refugiadas que tenían experiencias horrorosas de tortura y trauma. Recuerdo estar día tras día sentada en mi automóvil llorando después de las entrevistas. Necesitaba tomar un descanso después de eso ... podría haber seguido muchos caminos, y seguí el camino de ... luz, supongo; y lo tomé con la investigación acerca de las ancianas (en Ashfield) (ej.: Wise 2005, 2010). Me sorprendía ver que la única narrativa sobre raza y relaciones interétnicas que escuchábamos era negativa, enfatizando conflicto, tensión y déficit. Mis experiencias en las redes de solidaridad me hicieron pensar que había otra historia que contar. 
A fines de los 1990s, el discurso sobre las relaciones raciales en la literatura de Australia, Reino Unido y Estados Unidos era bastante distópico. Primaba la idea de que todos los encuentros a través de la diferencia se caracterizaban por el racismo blanco. Pero yo había estado viendo, a través de las ancianas y las iglesias, que estaba sucediendo esto otro también. Entonces vivíamos en Ashfield y veíamos racismo, pero también relaciones y redes de vecindad, no solo entre residentes blancos y no blancos, sino también entre personas de diferentes ascendencias minoritarias. Esto se convirtió en mi proyecto postdoctoral.

Me cambié a Australian National University, al centro interdisciplinario Center for CrossCultural Research. Había mayormente antropólogos, pero también cineastas etnográficos, artistas e historiadores poscoloniales. Era un ambiente muy estimulante, rodeado de personas como Peter Read, Dipesh Chakrabarty y reconocidos cineastas etnográficos como David MacDougall. Fue un campo de entrenamiento extraordinario para mí como joven etnógrafa. Tuve el privilegio de estar involucrada en la serie de talleres de capacitación "Retos para realizar" del renombrado historiador del Pacífico, Greg Dening. Él nos llevaba a pasear por los bosques circundantes y nos desafiaba a sintonizar todos nuestros sentidos con lo que estábamos experimentando, y nos empujaba a encontrar formas de dar vida a lo sensorial en nuestra escritura y presentaciones académicas. Esto influyó en gran parte de mis primeros trabajos sobre multiculturalismo cotidiano.

Por esos días, el trabajo interdisciplinario era incentivado, pero más tarde con la introducción de rankings basados en disciplina, repentinamente se regresó a las disciplinas en Australia. Se hizo evidente que para conseguir un trabajo necesitábamos ceñirnos a una disciplina. Comenzamos a asistir a conferencias disciplinarias, pensando a cuál podríamos asociarnos. Obtuvimos el trabajo (académico) y nos reconstruimos como sociólogos. Pero he encontrado un espacio para trabajar a través de las disciplinas y esta sigue siendo mi disposición principal.

SV: Prestamos mucha atención a lo que está sucediendo a nuestro alrededor, y es ahí en parte donde han surgido las inspiraciones en cuanto a ideas de investigación. Un ejemplo es ese artículo que escribimos sobre el festival gay y lésbico Mardi Gras de Sydney (Velayutham y Wise, 2001). Una vez nos cruzamos con carteles promocionando la fiesta bailable Mardi Gras y vimos que los organizadores habían usado una imagen distorsionada e hiper-sexualizada del dios hindú elefante, Ganesh, para promocionar el evento. Ganesh se convertía en Gaynesh. Esto provocó mucha reacción entre los indios-australianos, quienes veían el acto como blasfemia. No expresaban abiertamente sentimientos anti-homosexuales u homofóbicos, pero, paradójicamente, se unieron a los grupos antigay y a los partidos conservadores para protestar en contra... Así que ese tipo de involucramiento con lo que estaba sucediendo en el debate sobre multiculturalismo en Australia a nivel cotidiano resultó en este artículo sobre apropiación cultural en sociedades multiculturales. 
AW: En términos más generales, en Australia no estamos limitados por disciplinas, no estamos preguntando “qué dicen los sociólogos?” Más bien, nos centramos en un tema. Los estudios migratorios son generalmente un campo muy interdisciplinario. Supongo que eso también nos ha ayudado a comprender que no estamos reproduciendo ideas, como sucede cuando estás atrapado en tu propia comunidad disciplinaria.

\section{Sobre multiculturalismo cotidiano y convivencia}

CR: Eso se relaciona con mi próxima pregunta. Podrían contarnos acerca de su enfoque etnográfico "en terreno" (on-the-ground) a la experiencia de vivir con la diversidad ¿cómo llegaron a trabajar en él y cuál su contribución?

AW: Creo que proviene de un momento histórico. El interés en etnografía fundamentada (grounded ethnography) comenzó realmente alrededor de 2003, 2004 en adelante y luego se convierte en un lugar de interés común desde, aproximadamente el 2005. A veces, cuando un trabajo es criticado, ese momento académico o contexto se pierde ... Si bien no está saturado, es un campo muy rico ahora, así que estoy volviendo a mirar la dinámica entre lo estructural y lo cotidiano.

SV: Tras nuestro artículo del Mardi Gras, y siguiendo la investigación de Amanda en "Zonas de Contacto", comenzamos a pensar en establecer el campo del multiculturalismo cotidiano como metodología y como enfoque para el estudio de las experiencias vividas de comunidades culturalmente diversas. Sentíamos que gran parte de la investigación temprana sobre multiculturalismo en Australia adoptaba un enfoque desde arriba (topdown) y se centraba en las políticas de inmigración o asentamiento, y en los impedimentos institucionales/estructurales que enfrentan las comunidades migrantes recién llegadas. Nuestro objetivo era contar una historia diferente, es decir, "desde abajo" (bottom-up). Queríamos explorar cómo las personas de orígenes culturalmente diversos se llevaban bien, cuáles eran los facilitadores, etc. Y cuáles eran los puntos de tensión, conflicto y diferencia irreconciliables. Amanda y yo organizamos una conferencia sobre multiculturalismo cotidiano en el 2005, la cual luego llevó a nuestra publicación sobre este tema (Wise y Velayutham, 2009), cuando estábamos en el Centre for Research on Social Inclusion (en Macquarie University).

AW: En el contexto australiano, el multiculturalismo siempre había sido terreno de la sociología tradicional y de los paradigmas "desde arriba" (top-down): teorías políticas del multiculturalismo, el estudio del asentamiento a través de clásicos indicadores de integración. La cuestión relacional no existía como parte de la conversación multicultural. La pregunta por lo relacional siempre se entendió a través del paradigma del racismo. Entonces, quizás mirar desde un lente más antropológico durante mi doctorado giró mi atención a cuestiones de formación comunitaria y cultural... a cómo describir estos encuentros y relaciones que están aconteciendo. 
Estábamos empezando a pensar en formación de comunidad y en cómo un lente antropológico puede ayudar a describir prácticas cotidianas, rituales y reciprocidad ... La mirada antropológica te sintoniza con la emergencia de algo nuevo. Entonces giramos desde el estudio de espacios transaccionales del multiculturalismo a espacios relacionales vecinales. Cuando la mirada es discursiva o centrada en un problema, se filtran muchas cosas.

CC: En este contexto, ¿qué significa la convivencia para ustedes, si pudieran resumirlo para aquellos que no están familiarizados con el término? ¿Cómo los contextos geográficos particulares que han estado estudiando (Australia y Singapur) configuran, o no, sus formas de entender o abordar la convivencia?

SV: Volviendo al punto del intento por comprender las realidades cotidianas vividas de diversidad, que nos llevaron a pensar en las diferentes maneras en que las personas se involucran, interactúan y negocian la diferencia, quisiera complementar la información. En ese momento, había investigación académica en el Reino Unido y Australia sobre el racismo que era estructural y no tanto sobre lo cotidiano; era más bien acerca de las diferentes formas de racismo experimentadas por los migrantes y grupos minoritarios. Estábamos empezando a mirar dónde tiene lugar la diversidad productiva (positiva) y cómo se lleva a cabo. Eso nos llevó a reflexionar acerca de mi propia experiencia de haber vivido en Singapur y también en Australia, viviendo en barrios muy diversos. ¿Cuáles son los puntos de partida para que este tipo de relaciones vecinales positivas de vivir juntos o menos antagonistas tengan lugar?

Estas interacciones podrían no estar llevándonos a una amistad a largo plazo, o a fuertes relaciones vinculantes. Podría conducir solo a un sentido de civilidad, que a veces se establece a través de formas de reciprocidad, prácticas de dar obsequios... Y por eso tratamos de expandir ampliamente la noción de convivencia. Podría ser un vecino o vecina dándote algo de fruta que cosechó en su jardín, un plato cocinado o simples actos como saludar, hacer contacto visual y asentir con la cabeza (como saludo); breves momentos que consideramos bastante interesantes en sí mismos. Superponer a esto la micro-sociología de Goffman nos ayudó a pensar en cómo el suburbio y ciertos elementos pueden unir a las personas. Así es como comenzamos a enfocarnos en verdaderas experiencias vividas (real lived experiences).

Ciertamente no estamos pasando por alto o ignorando comportamientos y prácticas discriminatorias, pero como parte de la pregunta de investigación, especialmente como parte de la pregunta por lo cotidiano, queríamos investigar cómo las personas y nosotros mismos negociamos la diferencia cotidiana.

AW: Convivencia (Conviviality) - no usamos el sentido en inglés de la fiesta de convivencia, hospitalidad feliz, sino en el sentido que tiene en castellano la noción de convivir; convivencia, que significa vivir juntos, la práctica de vivir juntos. Estamos muy 
influenciados por nuestra inmersión en una importante línea de pensamiento. Ghassan Hage (2003) ha sido realmente importante, particularmente su primer trabajo Bourdiesiano sobre esperanza y capacidades. Y Greg Noble, quien ha escrito acerca del trabajo encarnado de construir comunidad (Noble, 2009). Entonces, cuando pensamos en la convivencia, comenzamos a ver el trabajo encarnado involucrado en la creación de una nueva comunidad. Una vez más, está esa mirada antropológica hacia prácticas de reciprocidad, lo ritual y el afecto. Y así comenzamos a ver la reciprocidad como un acto encarnado, que te saca de tu zona de confort para tratar con personas que son cultural y lingüísticamente diferentes a ti. Hay trabajo involucrado en eso: ¿Cómo encuentro la palabra adecuada o el modo de comunicación correctos? Entonces, cuando ustedes (en Chile) hablan de cómo los trabajadores latinoamericanos interactúan con los empleadores chinos, vemos que hay una gran cantidad de trabajo y esfuerzo en esa relación (Chan et al., 2019; Ramírez y Chan, 2018). La relación puede haber comenzado como algo transaccional, pero las personas están tratando de resolver distintas cosas.

Supongo que, en términos de Bourdieu, las formas de habitus que están incrustadas en relaciones de poder enfatizan cómo un escenario de negociación de diferencias conjuga un cierto habitus y una forma de ser. Entonces, una de las contribuciones que Greg Noble hizo a la literatura es su crítica de que Bourdieu realmente no explica cómo se origina el habitus. Bernard Lahire, también un Bourdieusiano, escribe sobre múltiples campos sociales. En sociedades multidimensionales complejas, nunca estamos realmente involucrados en un solo campo social. Entonces, si tú piensas en entornos multiétnicos, hay capas superpuestas en la adquisición de capacidades y nuevas formas de ser. En este sentido la convivencia resalta la idea de convivencia como trabajo y práctica que es productiva y finalmente encarnada. Sin embargo, este trabajo también incluye cuestiones de poder, jerarquías y todo eso; no todo es una feliz coexistencia. Pero, como dice Paul Gilroy (2004), este tipo de comunidades urbanas diversas son de igual modo logros sociales importantes y profundos. Puede tratarse de las formas 'más felices' de coexistencia, y eso es genial, pero parte del proyecto es también comprender qué entornos crean algo que funciona o algo que se desmorona, cuándo (comunidad) es frágil o cuándo la coexistencia se incrusta tan profundamente al punto que podríamos describirla como una disposición compartida o comunal. Recientemente hemos estado escribiendo sobre la convivencia lingüística en el multiculturalismo cotidiano, observando culturas de chistes y bromas en lugares de trabajo en Australia y Singapur. Anteriormente hemos escrito sobre relaciones alrededor de la comida y vecinales. Hay muchas maneras de abordar esta pregunta por la coexistencia y la convivencia (convivial coexistence).

CC: Reflexionando en lo que han visto durante su visita a Santiago ¿Qué podría contribuir el caso de Santiago a la comprensión de convivencia en un contexto más amplio de 
diversidad? ¿Qué encontraron de particular o común en relación con los contextos que han estado investigando?

SV: La diferencia más obvia para mí sería el tipo de diversidad o el nivel de diversidad que he visto en Santiago. Nuestros otros contextos de investigación se ajustan al marco de "superdiversidad" (Vertovec, 2007) con personas de todo el mundo en un mismo lugar. Siendo un extraño aquí, puedo ver algunas comunidades diversas visibles, como los haitianos, indios, chinos y coreanos. En cuanto a las otras nacionalidades/grupos étnicos (por ejemplo, peruanos, colombianos, bolivianos, argentinos, etc.) presentes en Chile, no puedo diferenciarlos. Pero supongo que los chilenos en general pueden identificar si alguien es de un país vecino en función de su apariencia o la forma en que hablan español. En cierto sentido, creo que la diversidad latina es menos problemática debido a las historias coloniales compartidas y la lingua franca (a excepción de los brasileños). Los indios, chinos y coreanos aquí están trabajando principalmente en tiendas y locales de comida, así que, en primera instancia, la relación con los locales es una especie de interacción transaccional. Estoy pensando en Simmel aquí. Los chinos, en particular, están involucrados en la gama baja de la globalización (low-end globalization): la venta de productos para el hogar y vestuario importado y barato. También emplean a chilenos como trabajadores en las tiendas. Yo he examinado el multiculturalismo cotidiano principalmente en el contexto anglófono y, por lo tanto, es interesante para mí ver cómo estos grupos que no hablan español se comunican con los locales. ¿Qué estrategias adoptan? Los haitianos, por otro lado, están principalmente en ocupaciones de bajos salarios. Como minorías migrantes visibles, la diferencia racial se destaca. Aquí es donde surge el desafío: la voluntad de los chilenos de abrazar y llevarse bien con el Otro racial.

CC: Lo que te oigo decir es que tu posicionalidad también afecta tu modo de ver diversidad.

SV: Correcto. Si uno es de origen no-europeo (incluidos los chilenos indígenas), su raza/etnia se hace evidente. Para ellos y para mí como indio-tamil, mi posición de identidad informa el modo en que me relaciono con la diversidad y el sentirme (o no) en casa. En contraste, lugares como Sidney y Londres, que tienen historias más largas de migración y presencia de comunidades asiáticas y afrodescendientes, tienden a producir una sensibilidad cosmopolita, donde uno se siente cómodo con la diversidad.

AW: ... es una cuestión de legibilidad. Lo que ves, lo que se convierte en algo significativo y por qué. Pienso que es una interesante herramienta de comparación; la pregunta por la legibilidad y qué significa. Además, no creo que sólo los lugares con una gran diversidad, o superdiversos, pueden enseñarnos algo acerca de la coexistencia. Creo que estudiar lugares de diversidad emergente tiene un gran valor, donde los marcos a través de los cuales organizamos la diferencia social y cultural - tanto discursiva, estructural como 
institucionalmente - no están aún totalmente imbricados; o donde la naturaleza y cualidad de la diversidad (generacional, o por país de origen, o paisajes y cambiantes conectividades sociopolíticas) están reorganizando las cosas de nuevas maneras.

SV: Lo cual nos lleva a la pregunta por la historia y política de los lugares. En el contexto asiático, siendo yo asiático-singapurense, había sido capaz de decir que la política de Estado es racializada, y en ella se superponen categorías de migrantes, países de procedencia, tipos de visa, etc. Todos estos ponen a locales y migrantes en una jerarquía desde la que puedo dilucidar cuáles serían las dinámicas locales en términos de interacciones, la forma de trabajar y lugar de residencia. Probablemente sea lo mismo aquí también.

AW: La pregunta más obvia es cómo esta fase muy rápida de multiculturalismo o diversidad (en Chile) se desplegará en un lugar que ya cuenta con cierta diversidad; con la cuestión indígena, siendo una sociedad de asentamiento colonial. No es como mirar un lugar como Japón que tiene un multiculturalismo emergente, pero en un ambiente muy homogéneo. ¿Qué significa eso para una nación como Chile que no ha tenido un concepto oficial de multiculturalismo o de lo multiétnico (incluyendo migración)? Es muy emergente, y es interesante observar a dónde irá todo eso.

En términos de mi propio trabajo, he estado muy inmersa en la literatura sobre globalización desde abajo e informalidades, por lo que ver eso en acción (en barrio Meiggs, Santiago, Chile) es realmente interesante. Siempre hemos visto redes interétnicas, ciertamente en Singapur, por lo que es bastante familiar ver a chinos trabajando con indios o malayos o viceversa... y también en Sidney. Y, por supuesto, hay una extensa literatura sobre diásporas minoritarias de intermediarios (middle-man minorities) como los chinos en el contexto del sudeste asiático. Pero ver esto en un contexto muy diferente como el chileno, donde las cosas están sucediendo rápidamente y con una gran concentración geográfica, es bastante interesante. Incluso la forma en que las informalidades median esta multiculturalidad emergente y configuran las relaciones interétnicas cotidianas de poder... Esto es diferente en Australia, donde no encuentras comercio callejero y otro tipo de informalidad, por lo que se produce un tipo de relación diferente.

Y otro punto es la cuestión mapuche en lo que refiere al multiculturalismo ${ }^{1}$ Eso es algo en lo cual podemos pensar (comparativamente) en términos del contexto australiano, ciertamente. Algo más de lo cual hemos estado hablando (durante nuestra visita a Chile) es

1 Los mapuches son una colectividad indígena del sur de Chile que históricamente, hasta el presente, han tenido una relación conflictiva con el Estado Chileno. Al momento de esta entrevista, había numerosas manifestaciones en el país debido a la muerte de Camilo Catrillanca, un joven mapuche que fue asesinado por un comando policial antiterrorista. Este caso intensificó las sostenidas críticas dirigidas al gobierno y la policía civil (carabineros), dada la represión y abuso sistemático hacia las comunidades indígenas en el sur de Chile. 
la cuestión de la Cuenca del Pacífico y todas sus islas ... La historia de Chile que en cierto sentido es como una isla circundada por el mar y las montañas, y Australia es algo así como una nación isleña y Singapur también. Todas son sociedades de asentamiento colonial. Además, debido a que Australia tiene una larga historia de multiculturalismo y grandes comunidades, como la comunidad china que ha estado desde el principio, hay menos relaciones comerciales fuera del grupo, algo que ha sido interesante ver en Chile. La comunicación interétnica es como una especie de necesidad, de llevarse bien entre sí, y eso está produciendo interesantes dinámicas... mientras que en Australia, la diáspora (china) ya es enorme, comunidades como la de los nuevos inmigrantes chinos en Australia, generando un mundo empresarial bastante autónomo. Eso es interesante, creo. Dado que las comunidades migrantes aún son pequeñas (en Chile) necesitan tener este alto nivel de interdependencia (Ramírez y Stefoni, 2019), porque (la diversificación) está teniendo lugar rápidamente y concentrada en vecindarios.

\section{Desafíos metodológicos y reflexiones en investigación}

CR: Una característica importante de su trabajo ha sido la colaboración de largo plazo ¿Qué significa la colaboración para ustedes - en términos prácticos, personales, éticos, políticos particularmente dadas sus diferentes posicionalidades en Singapur y Australia? ¿Qué posibilita y qué desafíos implica la colaboración en su experiencia?

SV: probablemente tenemos diferentes opiniones acerca de esto. Pero creo que compartimos ideas y valores respecto del tipo de mundo en que queremos o aspiramos a vivir: una sociedad acogedora y hospitalaria; desafiar estereotipos, prácticas discriminatorias y trabajar a través de los conflictos y tensiones que surgen entre personas con ascendencias culturales diversas que comparten un ritmo común.

AW: ... no estoy segura. Nuestra colaboración probablemente comenzó bebiendo [risas]. Todos éramos parte de un grupo de doctorado y salíamos a observar el mundo, y algunos de los primeros artículos surgieron de las notas que tomábamos al reverso de una servilleta con una cerveza; muchas largas conversaciones en grupo reducidas en un papel. Además, Raj (Selvaraj) creció en Singapur, y habíamos estado yendo y viniendo a Singapur desde Australia por un tiempo. Comenzamos a pensar en Australia a través de Singapur, lo que nos dio una perspectiva diferente sobre la coexistencia, la raza, etc. Raj también se unió un poco a mi investigación postdoctoral (en Ashfield), y vivíamos en el área, por lo que hablábamos de lo que estábamos viendo en nuestro vecindario.

(La colaboración) pasó a ser algo pragmático una vez que tuvimos hijos. Supongo que es una cosa de género, común para mujeres que trabajan... Muchas carreras profesionales de mujeres se detienen cuando llegan los niños, así que nos preguntamos cómo combinar familia e investigación, y cómo mantener los vínculos de Raj con Singapur. Pero hemos sido muy cuidadosos, manteniendo parte de nuestro trabajo separado también, lo cual es 
saludable. Raj tiene su propia carrera académica, por separado. Algunos de los otros trabajos fueron acerca de nosotros tratando de resolver cosas en nuestras propias vidas, como el trabajo acerca de la diáspora tamil, que refiere a la comunidad de Raj. Tratábamos de comprender lo que estábamos viendo y lo que estaba sucediendo; lo que esa antropología clásica desde "afuera" no lograba capturar.

Otra cosa es que siempre logramos una perspectiva desde dentro/desde fuera, sea lo que sea que estemos haciendo. Si estamos en Singapur, veo cosas acerca de Singapur que él no ve, o él está viendo cosas acerca de Singapur que yo no estoy viendo. Él comprende mucho mejor las diferencias más finas y sensibilidades culturales que yo paso por alto. Así mismo en Australia, él tiene la habilidad de conectar con las personas. Tiene esta suerte de hermandad, con la que él simplemente se aventura a hablar con las personas... Él tiene esta sensibilidad Jurong ${ }^{2}$ que trae de Singapur. Él puede estar en Australia y hablar a personas que son migrantes, o vendedores de la vía pública, o personas que son racialmente outsiders y ellos cómodamente hablan con él. Gran parte del tiempo, trabajando con trabajadores tamiles, usamos la traducción de interesantes maneras. Él hacía entrevistas en tamil y yo trataba de que traduzca conceptos para mí que quizás no calzan. Y el participante trataba de ayudarnos a encontrar la palabra correcta. Así capturamos las cosas más allá de las palabras en cierto modo, cuando escribimos acerca de afecto... entonces puedes hacer eso interlingüísticamente mejor de lo que puedes a través de un idioma. Te ayuda a superar categorías. Y volvimos a la pregunta por la legibilidad; comprender el ritual que está más allá de lo legible.

CR: ¿Y respecto de los desafíos u obstáculos de colaborar?

AW: Mientras hacemos lluvia de ideas, le damos forma a las ideas verbalmente y yo puedo poner las cosas en forma escrita. Pero la autoría... tenemos un acuerdo tácito de que cuando estamos escribiendo acerca de Asia, Raj es el primer autor, y si es acerca de Australia, yo soy la primera autora. Si es algo comparativo es un poco más complejo: es quien haya hecho el trabajo más conceptual, diseñando el artículo... Fuera de eso, necesitamos tener trabajo separado también, así podemos sentarnos con nuestras propias ideas.

Si vemos una película, yo estaré mirando la narrativa más amplia, y él estará diciendo “¿Viste ese letrero en el fondo?” O, en el campo, él encuentra detalles realmente interesantes... yo estaré interesada escuchando los intercambios entre un vendedor y un cliente, o mirando interesantes encuentros entre las personas que están a mi alrededor. Y luego nos sentamos y bebemos y comemos y conectamos lo que estamos viendo con los debates más amplios con los que estamos comprometidos. Pensando en eso, Singapur en una gran influencia a nivel cotidiano, lo cual es irónico porque desde afuera es visto como "Singapur multiétnico". Es un espacio de interacción tan rico en mezcla lingüística y cultural. Creo que fue Katherine Robinson quien describía esto como 'pensándonos a

2 Un área de Singapur donde Velayutham creció. 
nosotros mismos a través de otros lugares' (thinking ourselves through elsewheres). Para mí Singapur fue realmente útil en quebrar esa mirada, en cierto modo. Pensar lugares a través de otros lugares, eso ha sido influyente, abriendo nuestros ojos.

CR: Pensando acerca del trabajo comparativo que han realizado, como comparar procesos de convivencia en Sidney y Singapur ¿cómo su trabajo difiere o resuena con otras aproximaciones comparativas y supera el nacionalismo metodológico?

SV: En cierto modo, es difícil escapar del nacionalismo metodológico porque tenemos que capturar la especificidad de cada contexto. Tenemos que interpelar a aquellas grandes instituciones sistémicas, pilares estructurales que encontramos en las sociedades. Pero quizás para nosotros, al menos con (el proyecto) "Diversidad Cotidiana en el Lugar de Trabajo" (Everyday Diversity at the Workplace), la idea ha sido no remover el panorama más amplio, sino unir aquello con otras interacciones y conceptos como lugar de trabajo, humor, reciprocidad, intercambio de códigos, uso de diferentes lenguajes, y así... Hay temas en común en ambos lugares. De algún modo la narrativa más amplia podría ser similar por así decir, como los cambios en los lugares de trabajo neoliberales que están sucediendo en ambos países y que tienen consecuencias en como las personas trabajan e interactúan en el trabajo.

AW: Hemos estado muy profundamente inmersos en preguntas acerca de comparación, y nacionalismo metodológico, como Glick Schiller y otros (Wimmer y Glick Schiller, 2002). Ellos contribuyen con una comparación relacional antes que una comparación compartimentalizada, y una comparación temática en lugar de una basada en la nación. Pero también estamos influenciados por la sociología postcolonial - globalizaciones conectadas y marxismos reconstruidos. Pensando a través de historias coloniales acerca de extracción y explotación, una vez que logras una mirada global, pasas lo comparativo y comienzas a ver tejidos de conexiones. Esto ha sido importante, de la mano del seminal ensayo de Doreen Massey (1991), “A Global Sense of Place”, que en forma muy apta describe cómo moverse a través de los bordes nacionales no implica necesariamente comparación. Los estudios transnacionales nos sintonizan con eso.

Raj y yo estamos crecientemente pensando la multiculturalidad cotidiana y la diversidad urbana a través de la materialidad del espacio urbano. Esto implica pensar acerca de las materialidades y fuerzas que median la convergencia en la convivencia cotidiana: a quiénes juntan estas fuerzas, qué posibilita (o inhibe) estos procesos, qué tipos de convivencia, aunque sea circunscrita, emergen. Si bien han de parecer ciudades extrañas a comparar, en realidad hay muchas conexiones, tanto presentes como extendiéndose hacia el periodo colonial que continúa dando forma a la organización social de la diferencia. 


\section{Referencias bibliográficas}

Chan, C., Ramírez, C. y Stefoni, C. (2019). Negotiating Precarious Labour Relations: Dynamics of Vulnerability and Reciprocity between Chinese Employers and Their Migrant Workers in Santiago, Chile. Ethnic and Racial Studies 42 (9), 1456-1475.

Gilroy, P. (2004). After Empire: Melancholia or Convivial Culture? London: Routledge.

Hage, G. (2003). Against Paranoid Nationalism: Searching for Hope in a Shrinking Society. Pluto Press.

Massey, D. (1991). A Global Sense of Place. Marxism Today 38, 24-29.

Noble, G. (2009). Everyday Cosmopolitanism and the Labour of Intercultural Community. En A. Wise y S. Velayutham (eds.), Everyday multiculturalism (pp. 46-65). Palgrave Macmillan.

Ramírez, C. y Chan, C. (2018). Making Community under Shared Conditions of Insecurity: The Negotiation of Ethnic Borders in a Multicultural Commercial Neighbourhood in Santiago, Chile. Journal of Ethnic and Migration Studies, 1-18. https://doi.org/10.1080/1369183X.2018.1497953

Ramírez, C. y Stefoni, C. (2019). Contested and Interdependent Appropriation of Space in a Multicultural Commercial Neighbourhood of Santiago, Chile. Identities, 1-20. https://doi.org/10.1080/1070289X.2019.1658394

Velayutham, S. y Wise, A. (2001). Dancing with Ga (y) nesh: rethinking cultural appropriation in multicultural Australia. Postcolonial Studies: Culture, Politics, Economy, 4(2), 143-160.

Vertovec, S. (2007). Super-diversity and its implications. Ethnic and Racial Studies 30 (6), 1024-1054. https://doi.org/10.1080/01419870701599465

Wimmer, A. y Glick-Schiller, N. (2002). Methodological nationalism and beyond: nationstate building, migration and the social sciences. Global Networks 2(4), 301-334. https://doi.org/10.1111/1471-0374.00043

Wise, A. (2006). Exile and return among the East Timorese. University of Pennsylvania Press.

Wise, A. (2005). Hope and Belonging in a Multicultural Suburb. Journal of Intercultural Studies 26 (1-2), 171-86. https://doi.org/10.1080/07256860500074383

Wise, A. (2010). Sensuous Multiculturalism: Emotional Landscapes of Inter-Ethnic Living in Australian Suburbia. Journal of Ethnic and Migration Studies 36 (6), 917937. https://doi.org/10.1080/13691831003643355

Wise, A. y Velayutham, S. (2009). Everyday multiculturalism. Palgrave Macmillan. 